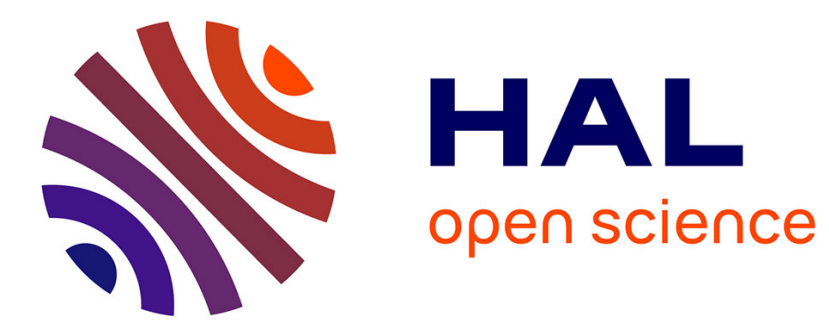

\title{
Simple example of fluctuation induced reentrant behaviour
}

C. Coulon, Jacques Prost

\section{To cite this version:}

C. Coulon, Jacques Prost. Simple example of fluctuation induced reentrant behaviour. Journal de Physique Lettres, 1981, 42 (11), pp.241-244. 10.1051/jphyslet:019810042011024100 . jpa-00231918

\section{HAL Id: jpa-00231918 https://hal.science/jpa-00231918}

Submitted on 1 Jan 1981

HAL is a multi-disciplinary open access archive for the deposit and dissemination of scientific research documents, whether they are published or not. The documents may come from teaching and research institutions in France or abroad, or from public or private research centers.
L'archive ouverte pluridisciplinaire HAL, est destinée au dépôt et à la diffusion de documents scientifiques de niveau recherche, publiés ou non, émanant des établissements d'enseignement et de recherche français ou étrangers, des laboratoires publics ou privés. 


\title{
Simple example of fluctuation induced reentrant behaviour
}

\author{
C. Coulon and J. Prost \\ Centre de Recherche Paul Pascal, Domaine Universitaire, 33405 Talence cedex, France
}

(Reçu le 17 novembre 1980, révisé le 23 mars 1981, accepté le 7 avril 1981)

\begin{abstract}
Résumé. - Nous montrons qu'un système quasi unidimensionnel décrit par deux paramètres d'ordre d'Ising couplés est susceptible de présenter un comportement rentrant. Quand les deux paramètres d'ordre ont la même " tendance » à condenser, on retrouve la phase haute température, celle-ci ayant pour origine des fluctuations importantes d'un des états condensés à l'autre.
\end{abstract}

\begin{abstract}
We show that a quasi-one dimensional system, described by two coupled Ising order parameters, can show a reentrant behaviour. The high temperature phase is recovered when the two order parameters have the same " tendency » for condensation and results from large fluctuations from one condensed state to the other.
\end{abstract}

1. Introduction. - The purpose of this note is to illustrate in a simple and analytically soluble example the idea [1], that, under certain conditions, a system may be unable to choose between two order parameters and revert back to the most symmetric phase, although each of these parameters would be condensed if independently considered. Indeed, renormalization group analysis gives [2] for a system in which two order parameters $\sigma_{1}, \sigma_{2}$ (dimensionality $n_{1}, n_{2}$ ) compete for condensation :

$$
\begin{gathered}
\left(T_{\sigma_{1}}-T_{\sigma_{1}}^{\mathrm{MF}}\right) \propto\left(n_{1}+2\right) ;\left(T_{\sigma_{2}}-T_{\sigma_{2}}^{\mathrm{MF}}\right) \propto\left(n_{2}+2\right) ; \\
\left(T_{\sigma_{1} \sigma_{2}}-T_{\sigma_{1} \sigma_{2}}^{\mathrm{MF}}\right) \propto\left(n_{1}+n_{2}+2\right) .
\end{gathered}
$$

$T_{\sigma_{1}}, T_{\sigma_{2}}$ are respectively the $\sigma_{1}$ and $\sigma_{2}$ condensation temperatures; $T_{\sigma_{1} \sigma_{2}}$ the simultaneous condensation temperature of $\sigma_{1}$ and $\sigma_{2}$; the superscript MF stands for mean field.

From these relations, it is clear that the most symmetric phase is stabilized when $\sigma_{1}$ and $\sigma_{2}$ condense together, which leads to the possibility of reentrant behaviour [1]. However, the difference $T-T^{\mathrm{MF}}$ is not very easy to calculate accurately, due in particular to its non universality [3]. Thus we find it useful to consider the simple case of two coupled Ising order parameters in a quasi-one dimensional system, because fluctuations may be accounted for, rigorously in one dimension through the transfer matrix technique [4], and with appropriate approximations in nearly one dimensional systems [5]. In particular, transition temperatures are easy to calculate with this method and we are indeed able to show the possibility of (doubly) reentrant behaviour.
Finally, we point out that quasi-one dimensional systems exist in nature, which gives more relevance to our example [6]. A particularly interesting case, although slightly different, is provided by the series of TMTSF salts, in which a competition between superconducting and spin density wave states is known to occur [7].

2. Description and resolution of the model. - We consider a set of chains, weakly correlated with each other, in which two Ising order parameters, $\sigma_{1}$ and $\sigma_{2}$ are susceptible to condensation. For pedagogical simplicity, we assume that $\sigma_{1}$ and $\sigma_{2}$ have different symmetries. The free energy per chain may be written

$$
\begin{aligned}
2 F=\int_{0}^{L} \mathrm{~d} z\left[A_{1}\right. & \sigma_{1}^{2}+A_{2} \sigma_{2}^{2}+C_{\|} \times \\
& \left.\times\left(\left(\nabla_{z} \sigma_{1}\right)^{2}+\left(\nabla_{z} \sigma_{2}\right)^{2}\right)+\frac{1}{2} B_{i j} \sigma_{i}^{2} \sigma_{j}^{2}\right] \\
& -\int_{0}^{L} \sum_{n=1}^{n=Z} \sigma_{i} \sigma_{j n} C_{i j} \mathrm{~d} z
\end{aligned}
$$

$A_{1}$ and $A_{2}$ are the usual coefficients of the harmonic terms, which change sign at the mean field transition temperatures. $(i, j) \in(1,2)$; the sum over $n$ extends over the $Z$ first nearest neighbours of a given spin $i$. $C_{i j}$ is the matrix of the transverse coupling coefficients. $C_{i j}$ is necessarily diagonal but can be anisotropic in the $\sigma_{1}, \sigma_{2}$ space. The choice of a unique elastic constant $C_{\|}$can always be made through a redefinition of $\sigma_{1}$ and $\sigma_{2}$. 
This free energy has been investigated by Imry et al. [8] in the purely one dimensional case for which there is no possibility of finding any reentrant behaviour since there are no phase transitions in 1d systems. The influence of the $B_{i j}$ anisotropy, in the case $A_{1}=A_{2}$ has been studied by Lederer and Pitanga [9]. The $n=1$ to $n=2$ cross-over was observed, but since fourth order coefficients are usually temperature independent, again no reentrant phenomenon was hinted at. On the other hand, the microscopic model for ferroelectric behaviour of Tornau and Shneider contains the possibility of reentrance [10] but without any general discussion of its origin. The merit of our approach is to give a simple and general interpretation of this unusual behaviour, in terms of a competition between $\sigma_{1}$ and $\sigma_{2}$. In order to describe this competition, we introduce different $A_{1}$ and $A_{2}$ which have the same value only at a given temperature $T_{A A}$ (see Fig. 1a). For a weak fourth

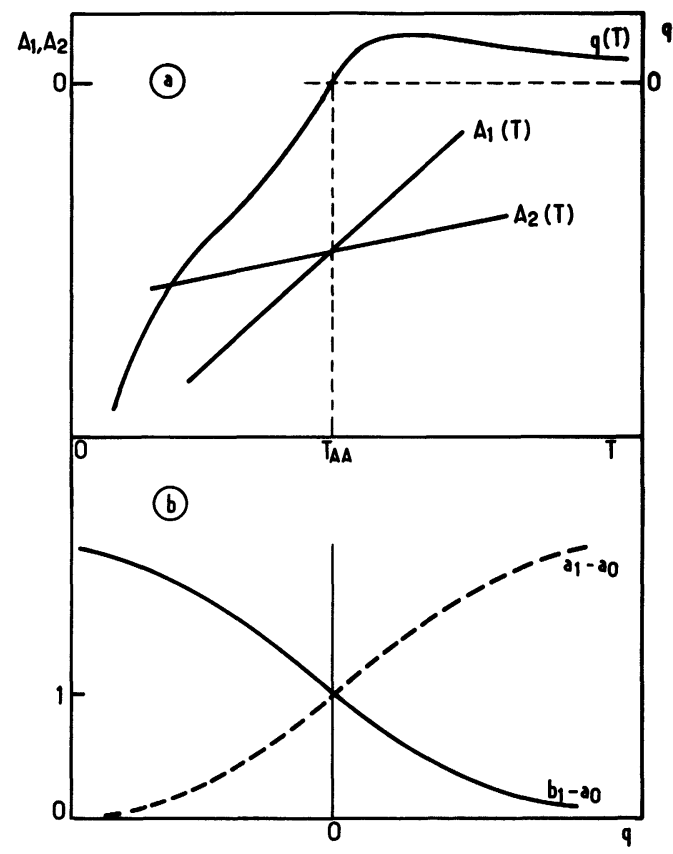

Fig. 1. - a) Temperature dependence of $A_{1}, A_{2}$ and $q . T_{A A}$ is defined by $A_{1}\left(T_{A A}\right)=A_{2}\left(T_{A A}\right)$. b) $q$ dependence of $b_{1}-a_{0}$ (full line) and $a_{1}-a_{0}$ (dashed line) according to reference [11]. Note the crossing of the levels at $q=0$.

order anisotropy, the system should behave as a one component Ising system except in the vicinity of $T_{A A}$ where the $n=2$ behaviour is expected according to [9]. For analytical simplicity, we will consider isotropic fourth order terms only $\left(B_{11}=B_{22}=B_{12} / 2=B\right)$. (It is possible to check, by perturbation techniques, that the results we will derive are not qualitatively altered by small anisotropies.)

With the change of variable :

$$
\begin{array}{ll}
\sigma_{1}=\sigma \cos \theta & \gamma=\left(A_{1}+A_{2}\right) / 2 \\
\sigma_{2}=\sigma \sin \theta . & \alpha=\left(A_{1}-A_{2}\right) / 2 .
\end{array}
$$

One finds for the one dimensional part of the free energy :

$$
\begin{aligned}
2 F_{1}=\int_{0}^{L} \mathrm{~d} z\left[\gamma \sigma^{2}+\right. & \frac{B}{2} \sigma^{4}+\alpha \sigma^{2} \cos 2 \theta+C_{\|} \times \\
& \left.\times\left(\sigma^{2}\left(\nabla_{z} \theta\right)^{2}+\left(\nabla_{z} \sigma\right)^{2}\right)\right]
\end{aligned}
$$

The evaluation of the 1d partition function may be obtained with the use of the transfer matrix technique. It requires the solution of an associated quantum mechanical problem :

with :

$$
\mathscr{H}_{0} \Psi_{n}=\varepsilon_{n} \Psi_{n}
$$

$$
\begin{aligned}
& \mathscr{H}_{0}=-\frac{1}{2 m^{*}} \Delta+\gamma \frac{\sigma^{2}}{2}+B \frac{\sigma^{4}}{4}+\frac{\sigma^{2}}{2} \alpha \cos 2 \theta \\
& \frac{1}{m^{*}}=\frac{k_{\mathrm{B}}^{2} T^{2}}{C_{\|}} \quad \Delta=\frac{\partial^{2}}{\partial \sigma^{2}}+\frac{1}{\sigma} \frac{\sigma}{\partial \sigma}+\frac{1}{\sigma^{2}} \frac{\partial^{2}}{\partial \theta^{2}} .
\end{aligned}
$$

This is nothing but the vibration-rotation Hamiltonian for which there is an analytical solution when a small hindrance may be assumed. This is the most interesting situation, i.e. the one which insures large fluctuations between $\sigma_{1}$ and $\sigma_{2}$. It corresponds to :

$$
|\gamma| \gg|\alpha| \text {. }
$$

We will focus our attention on this case in the following. Note further that, because of the importance of $1 \mathrm{~d}$ fluctuations, $\gamma$ is negative with a large absolute value in the temperature domain of interest. In other words, $\gamma$ is very close to its mean field value $\sigma_{0}=\left(-\frac{\gamma}{B}\right)^{1 / 2}$ and the first excited states of $\mathcal{H}_{0}$ arise from the angular perturbations of the fundamental mode of vibration. The relevant Hamiltonian is therefore :

$$
\mathscr{H}_{0}=-\frac{1}{2 m^{*} \sigma_{0}^{2}} \frac{\mathrm{d}^{2}}{\mathrm{~d} \theta^{2}}+\frac{1}{2} \sigma_{0}^{2} \alpha \cos 2 \theta .
$$

The corresponding eigenvalue equation is a Mathieu equation [11] of generic form :

$$
\left[\frac{\mathrm{d}^{2}}{\mathrm{~d} \theta^{2}}+a+2 q(1-\cos \theta)\right] \Phi=0
$$

with

$$
\begin{gathered}
q=m^{*} \sigma_{0}^{4} \alpha / 2 \\
\varepsilon_{i}=\frac{a_{i}}{2 m^{*} \sigma_{0}^{2}}+\frac{\sigma_{0}^{2} \alpha}{2} .
\end{gathered}
$$

The eigenvectors are called Mathieu functions, and usually denoted $\operatorname{ce}_{m}(\theta, q)$ or $\operatorname{se}_{m}(\theta, q)$ according to the symmetry of the function with respect to $\theta$. The corresponding eigenvalues are respectively called $a_{m}(q)$ and $b_{m}(q)$ [11]. For positive $q$, the first three levels are obtained with $\mathrm{ce}_{0}$ (ground state), $\mathrm{se}_{1}$ (first excited) and $\mathrm{ce}_{1}$ (second excited). For negative $q$, 
the sequence is changed : $c_{0}$ (ground state), ce $_{1}$ (first excited), $\mathrm{se}_{1}$ (second excited). The level crossing occurs for $q=0$ (note that, in this case, the degeneracy of the excited states is obvious from direct inspection of (5)). The eigenvalue differences $\left(a_{1}-a_{0}\right)$ and $\left(b_{1}-a_{0}\right)$ are displayed in figure 1 . Knowledge of the $\varepsilon_{i}$ through (6) allows the calculation of the $1 \mathrm{~d}$ correlation functions and $\varepsilon_{0}$ gives the 1d free energy [4].

There is indeed no phase transition in such a one dimensional problem, but the corresponding weakly coupled three dimensional system is known to exhibit a finite ordering temperature obtained with good accuracy from an appropriate mean field treatment of the interchain interaction [5]. In this approximation, the Hamiltonian corresponding to the $3 \mathrm{~d}$ problem reads :

$$
\mathscr{H}=\mathscr{H}_{0}-Z C_{i i} \sigma_{i} \bar{\sigma}_{i}
$$

where $\bar{\sigma}_{i}$ is the mean value of the order parameter $\sigma_{i}$.

Although this problem may usually be treated in the two level approximation, the level crossing for $q=0$ imposes the retention of three states of $\mathcal{H}$.

Using $\mathrm{ce}_{0}, \mathrm{se}_{0}, \mathrm{ce}_{1}$ as eigenstates and expanding the ground state $E_{0}$ of $\mathscr{H}$ in powers of $\bar{\sigma}_{1}$ and $\bar{\sigma}_{2}$ gives :

$$
\begin{gathered}
E_{0}=-\frac{\lambda_{-}^{2}\left(Z C_{11} \sigma_{0}\right)^{2}}{\varepsilon_{-}-\varepsilon_{0}} \bar{\sigma}_{1}^{2}-\frac{\lambda_{+}^{2}\left(Z C_{22} \sigma_{0}\right)^{2}}{\varepsilon_{+}-\varepsilon_{0}} \bar{\sigma}_{2}^{2}+ \\
+\frac{\lambda_{-}^{4}\left(Z C_{11} \sigma_{0}\right)^{4}}{\left(\varepsilon_{-}-\varepsilon_{0}\right)^{3}} \bar{\sigma}_{1}^{4}+\frac{\lambda_{+}^{4}\left(Z C_{22} \sigma_{0}\right)^{4}}{\left(\varepsilon_{+}-\varepsilon_{0}\right)^{3}} \bar{\sigma}_{2}^{4} \\
+\frac{\lambda_{+}^{2} \lambda_{-}^{2}\left(Z^{2} \sigma_{0}^{2} C_{11} C_{22}\right)^{2}\left(\varepsilon_{+}+\varepsilon_{-}-2 \varepsilon_{0}\right)}{\left(\varepsilon_{+}-\varepsilon_{0}\right)^{2}\left(\varepsilon_{-}-\varepsilon_{0}\right)^{2}} \bar{\sigma}_{1}^{2} \bar{\sigma}_{2}^{2}
\end{gathered}
$$

where :

$$
\begin{aligned}
& \lambda_{-}=\left\langle\mathrm{ce}_{1}|\cos \theta| \mathrm{ce}_{0}\right\rangle \\
& \lambda_{+}=\left\langle\mathrm{se}_{1}|\sin \theta| \mathrm{ce}_{0}\right\rangle
\end{aligned}
$$

$\varepsilon_{+}$and $\varepsilon_{-}$are the energy levels corresponding, respectively, to $b_{1}$ and $a_{1}$ and given by (6). (Note that because of the above mentioned level crossing, $\varepsilon_{+}$is the first excited state for $\alpha>0$, whereas it is $\varepsilon_{-}$for $\alpha<0$.)

The thermal dependence of $\lambda_{ \pm}$and $\left(\varepsilon_{ \pm}-\varepsilon_{0}\right)$, which results from the properties of the Mathieu functions and the non-monotonic temperature dependence of $|q|$ (Fig. 1) are given in figure 2. Note that the $\varepsilon_{+}-\varepsilon_{0}$ curve may or may not be monotonic.

With the self consistent field energy $\frac{1}{2} J C_{i i} \bar{\sigma}_{i}^{2}$, the free energy density reads :

$$
\begin{aligned}
\Delta F=\left[\frac{1}{2} Z C_{11}-\frac{\lambda_{-}^{2}\left(Z C_{11} \sigma_{0}\right)^{2}}{\varepsilon_{-}-\varepsilon_{0}}\right] \bar{\sigma}_{1}^{2}+ \\
+\left[\frac{1}{2} Z C_{22}-\frac{\lambda_{+}^{2}\left(Z C_{22} \sigma_{0}\right)^{2}}{\varepsilon_{+}-\varepsilon_{0}}\right] \bar{\sigma}_{2}^{2} \\
+\tilde{B}_{i j} \bar{\sigma}_{i}^{2} \bar{\sigma}_{j}^{2}
\end{aligned}
$$

where the $\tilde{B}_{i j}$ 's are the coefficients of the fourth order terms given by (8).

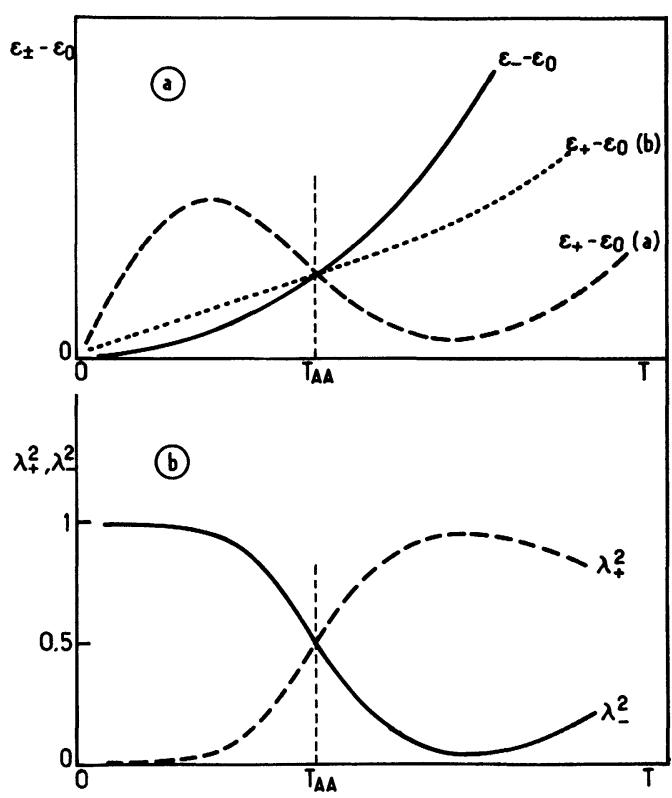

Fig. 2. $-a$ ) Temperature dependence of $\varepsilon_{-}-\varepsilon_{0}$ (full line), $\varepsilon_{+}-\varepsilon_{0}$ when $\frac{\mathrm{d} \alpha}{\mathrm{d} T}\left(T_{A A}\right)$ is large (dashed line), $\varepsilon_{+}-\varepsilon_{0}$ when $\frac{\mathrm{d} \alpha}{\mathrm{d} T}\left(T_{A A}\right)$ is small (dotted line). $b$ ) Temperature dependence of $\lambda_{-}^{2}$ (full line) and $\lambda_{+}^{2}$ (dashed line).

This free energy density is to be considered as a conventional Landau expansion in which, however, the coefficients have non trivial temperature dependences.

As a consequence of the anisotropy of the fourth order terms :

$$
\widetilde{B}_{12}^{2}=\widetilde{B}_{11} \tilde{B}_{22} \frac{\left(\varepsilon_{+}+\varepsilon_{-}-2 \varepsilon_{0}\right)^{2}}{\left(\varepsilon_{+}-\varepsilon_{0}\right)\left(\varepsilon_{-}-\varepsilon_{0}\right)}>\widetilde{B}_{11} \tilde{B}_{22}
$$

the only stable phases are :

$$
\begin{aligned}
& \mathrm{N} \bar{\sigma}_{1}=\bar{\sigma}_{2}=0 \text { the high temperature phase, } \\
& \mathrm{P}_{1} \bar{\sigma}_{1} \neq 0 \text { and } \bar{\sigma}_{2}=0, \\
& \mathrm{P}_{2} \bar{\sigma}_{1}=0 \text { and } \bar{\sigma}_{2} \neq 0 .
\end{aligned}
$$

The $N \leftrightarrow P_{1}$ and $N \leftrightarrow P_{2}$ transitions are second order. The transition temperatures are given respectively by :

$$
\begin{aligned}
& \tilde{A}_{1}=\frac{1}{2} Z C_{11}-\frac{\lambda_{-}^{2}\left(Z C_{11} \sigma_{0}\right)^{2}}{\varepsilon_{-}-\varepsilon_{0}}=0 \\
& \tilde{A}_{2}=\frac{1}{2} Z C_{22}-\frac{\lambda_{+}^{2}\left(Z C_{22} \sigma_{0}\right)^{2}}{\varepsilon_{+}-\varepsilon_{0}}=0 .
\end{aligned}
$$

The $P_{1} \leftrightarrow P_{2}$ transition is first order and occurs when :

$$
\frac{\tilde{A}_{1}^{2}}{\tilde{B}_{11}}=\frac{\tilde{A}_{2}^{2}}{\tilde{B}_{22}} \text { and } \begin{aligned}
& \tilde{A}_{1}<0 \\
& \tilde{B}_{2}<0
\end{aligned}
$$

The non trivial temperature dependence of the $\tilde{A}_{i}$ 's and $\widetilde{B}_{i i}$ 's comes from that of $\lambda_{ \pm}$and $\varepsilon_{ \pm}-\varepsilon_{0}$ (Fig. 2). We give in figure 3 three examples of deduced phase 
diagrams. The first two show the condition for reentrant behaviour (when $C_{11}=C_{22}$ ) : it depends on the sign of the slope of the $A_{2}\left(T, C_{22}\right)=0$ curve at $T_{A A}$ and the condition for the occurrence of reentrance is in this case

$$
\frac{3}{8} C_{\|} \gamma^{2} \frac{\mathrm{d} \alpha}{\mathrm{d} T}\left(T_{A A}\right)>k_{\mathrm{B}}^{2} B^{2} T_{A A}
$$

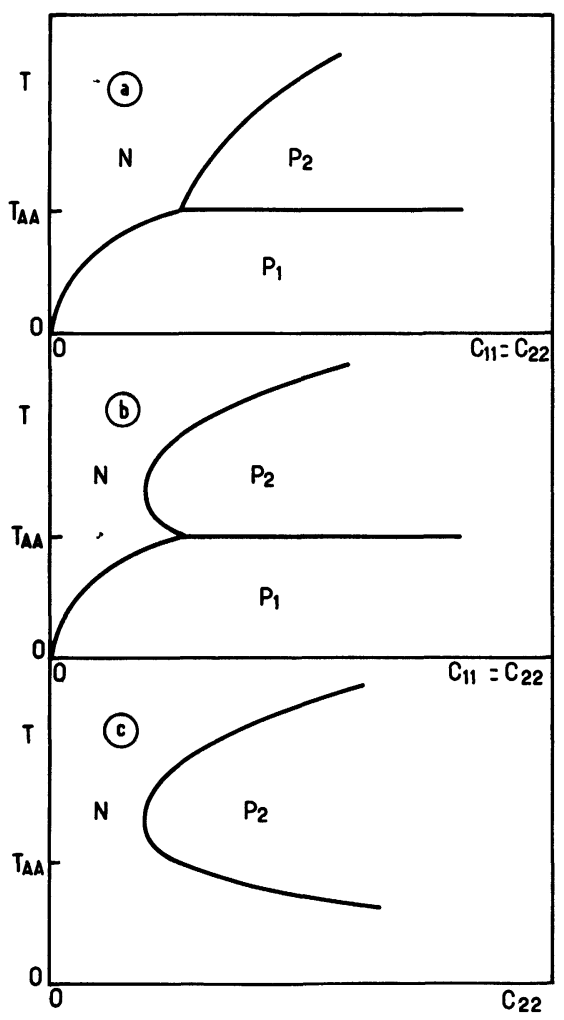

Fig. 3. - a) Phase diagram for $C_{11}=C_{22}$ and condition (13) not fulfilled. $b$ ) Phase diagram for $C_{11}=C_{22}$ and condition (13) fulfilled. $c$ ) Phase diagram for $C_{11}=0$.

The third example corresponds to $C_{11}=0$. It gives the largest reentrant domain, although the $\sigma_{1}$ order never condenses.
3. Concluding remarks. - There is indeed the possibility for two coupled Ising order parameters to show a reentrant behaviour. The origin of this phenomenon is precisely, as mentioned in the introduction, the competition between $\sigma_{1}$ and $\sigma_{2}$ in the $T_{A A}$ region :

- far from this point, only one of the order parameters fluctuates significantly; the 1d correlation length varies essentially as

$$
\left(\varepsilon_{1}-\varepsilon_{0}\right)^{-1} \propto \exp (4 \sqrt{|q|})
$$

which characterizes unambiguously a $n=1$ problem;

- on the other hand, in the neighbourhood of $T_{A A}$, both $\sigma_{1}$ and $\sigma_{2}$ fluctuate, the $1 \mathrm{~d}$ correlation length goes as $1 / T_{A A}$ which is typical of a $n=2$ condensation problem. Another even more transparent clue of the switch from $n=1$ to $n=2$, behaviour, in the reentrant domain, is the degeneracy of the excited states in the dual quantum mechanical problem exactly at $T_{A A}$.

Note that it is not necessary for $\sigma_{1}$ to condense in order to lead to a $\sigma_{2}$ reentrant behaviour. The case $C_{11}=0$, in which no $3 \mathrm{~d}$ ordering temperature exists for $\sigma_{1}$ exhibits at best the suppression of the $\sigma_{2}$ order by the $\sigma_{1}$ fluctuations (Fig. $3 c$ ) ; we think that this mechanism could explain the reentrant phases observed in some systems $[1,12,13]$ in which competition between two order parameters is known to exist. Our model is however only illustrative of the processes involved in such more complicated phases : for instance in the reentrant nematic phases, the order parameters are complex and incommensurability problems cannot be excluded. On the other hand, the quasi-one dimensional conductors of the TMTSF series might be a better example of the argument developed above. In any case, we consider this work as a pedagogical example in which the competition between two order parameters leads to a reentrant behaviour.

\section{References}

[1] Prost, J., in Proceedings of the conference on liquid crystal of one and two dimensional order, Garmish-Partenkirchen, (Springer-Verlag Berlin, Heidelberg, New York) 1980 , p. 125.

[2] Toulouse, G., Pfeuty, P., Introduction au groupe de renormalisation et à ses applications (Presses Universitaires de Grenoble) 1975.

[3] Wilson, K. G., Kogut, J., Phys. Rep. 12C, nº 2 (1974). Melting and other phase transitions in two dimensions excepted, See for instance :

Halperin, B. I., Nelson, D. R., Phys. Rev. Lett. 41 (1978) 121.

[4] Scalapino, D. J., Sears, M., Ferell, R. A., Phys. Rev. B 6 (1972) 3408

[5] Scalapino, D. J., ImRy, Y., Pincus, P., Phys. Rev. B 11 (1975) 2042 and

Dieterich, W., Adv. Phys. 25 (1976) 615.
[6] For example organic conductors such as TTF-TCNQ. For a review see :

Toons, G. A., Phys. Rep. C 40 (1978) 181.

[7] Parkin, S. S. P., Ribault, M., Jerome, D., Bechgaard, K., to be published.

[8] ImRY, Y., Scalapino, D. J., Gunther, L., Phys. Rev. B 10 (1974) 2900 and references therein.

[9] Lederer, P., Pitanga, P., J. Physique 39 (1978) 993.

[10] Tornau, E. E., Shneider, V. E., JETP Lett. 31 (1980) 357.

[11] See for example : Handbook of Mathematical functions, (Dover publications New York) 1964, p. 721.

[12] For a review of experimental work on doubly reentrant liquid crystal, see for instance :

Sigaud, G., Nguyen huU tinh, Hardouin, F., GaspaROUX, H., to be published in Mol. Cryst. Liq. Cryst.

[13] Delhaes, P., Coulon, C., Manceau, J. P., Flandrois, S., FaBre, J. M., Giral, L., to be published. 